\title{
POTENCIALES ÁREAS CULTIVABLES DE PASIFLORAS EN UNA REGIÓN TROPICAL CONSIDERANDO ESCENARIOS DE CAMBIO CLIMÁTICO
}

\author{
POTENTIAL SUITABLE AREAS FOR PASSIFLORAS CROPS IN A TROPICAL \\ REGION CONSIDERING CLIMATE CHANGE SCENARIOS
}

Andrés Mauricio Munar • mauriciomunar4@gmail.com

PhD en Recursos Hídricos y Saneamiento Ambiental, Corporación Centro de Desarrollo Tecnológico de las Pasifloras de Colombia [CEPASS], Neiva, Colombia.

Adalberto Rodríguez Carlosama•adalberto.rodriguez@cepass.org Magíster (C) en Ciencias Agrarias, Corporación Centro de Desarrollo Tecnológico de las Pasifloras de Colombia [CEPASS], Neiva, Colombia

Jorge Luis Muñoz España・jorge.munoz@cepass.org

Ingeniero Agrónomo, Corporación Centro de Desarrollo Tecnológico de las Pasifloras de Colombia [CEPASS], Neiva, Colombia

Citación: Munar, A., Rodríguez, A., Muñoz, J. (2022). Potenciales áreas cultivables de pasifloras en una región tropical bajo escenarios de cambio climático. Revista de Investigación Agraria y Ambiental, 13(1), 109 - 129. DOI: https://doi.org/10.22490/21456453.4637

\section{RESUMEN}

Contextualización: El cambio climático y las actividades antrópicas sobre los recursos naturales se constituyen como los principales causantes de la pérdida de biodiversidad y la redistribución de las especies.

Vacío de conocimiento: Sin embargo, los efectos a nivel de comunidades y ecosistemas, así como los impactos en cultivos agrícolas en escala regional, son poco estudiados. Los modelos de distribución de especies se han convertido, por lo tanto, en valiosas herramientas para la predicción de áreas potencialmente aptas para especies cultivables, su gestión y planificación.

Propósito: Este estudio consistió en la predicción de potenciales áreas cultivables de maracuyá (Passifora edulis var. favicarpa Degener), granadilla (Passifora ligularis Juss), y cholupa (Passifora maliformis L.) en una región tropical, a través del modelo MaxEnt, en escenarios de cambio climático.

Metodología: Se utilizaron como datos de entrada (para el modelo MaxEnt) registros de presencia de las especies analizadas, obtenidos a partir de sus coordenadas geográficas. En total, fueron usados 141 registros de presencia de maracuyá, 256 registros de granadilla y 40 registros de cholupa, así como 12 variables bioclimáticas para las proyecciones actuales y futuras en los periodos 2050 y 2070, considerando así dos escenarios RCPs (Representative Concentration Pathways) del Coupled Model Intercomparison Project (CMIP5) (RCP 4.5 y RCP 8.5).

Resultados y conclusiones: LoS resultados revelan que las potenciales áreas cultivables para las especies analizadas podrían pronosticarse a través de MaxEnt utilizando registros de presencia en campo y variables bioclimáticas. Así mismo, las simulaciones indicaron que las áreas de ocurrencia potencial para las especies analizadas podrían disminuir en el futuro dependiendo de los escenarios climáticos (RCP 4.5 y RCP 8.5) para los periodos 2050 y 2070. Para los cultivos de maracuyá, granadilla y cholupa, las mayores 
reducciones en las áreas de ocurrencia potencial corresponden al $23 \%$, $25 \%$ y $31 \%$ respectivamente $y$, se presentarían en el período 2070 en un escenario pesimista (RCP 8.5). Este es el primer estudio que pronostica las potenciales áreas cultivables de pasifloras utilizando el modelo Maxent y escenarios de cambio climático en escala regional en una región tropical. El abordaje propuesto puede proveer importantes herramientas para la gestión y aprovechamiento sostenible de las especies estudiadas.

Palabras clave: Passiflora edulis var. flavicarpa Degener, Passiflora ligularis Juss, Passiflora maliformis L., MaxEnt

\section{ABSTRACT}

Contextualization: Climate change and anthropic activities on natural resources are the main causes of the biodiversity loss and the species redistribution.

Knowledge gap: However, the effects at the community and ecosystem levels, as well as the impacts on agricultural crops on a regional scale, are little studied. Species distribution models have become valuable tools for the prediction of areas potentially suitable for cultivable species, their management and planning.

Purpose: This research pretends predict the potential of cultivable areas for passion fruit (Passifora edulis var. favicarpa Degener), grenadia (Passifora ligularis Juss), and sweet calabash (Passifora maliformis L.), in a tropical region, using the MaxEnt model and considering climate change scenarios.

Methodology: Occurrence records of the analyzed species, obtained from their geographic coordinates, were used as input data for the MaxEnt model. In total, 141 occurrence records of passion fruit, 256 records of grenadia and 40 records of sweet calabash were used for the MaxEnt model, as well as 12 bioclimatic variables for the current and future projections in the 2050 and 2070 periods, considering two Representative Concentration Pathways RCPs from the CMIP5 (RCP 4.5 and RCP 8.5)

Results and conclusions: The results revealed that the potential suitable areas for the analysed species could be predicted from the MaxEnt model, using field records and bioclimatic variables. Likewise, the simulations indicated that the areas of potential occurrence for the analysed species could decrease in the future, considering climatic scenarios (RCP 4.5 and RCP 8.5) for the periods 2050 and 2070. For the passion fruit, grenadia and sweet calabash crops, the greatest reductions in the potential suitable areas correspond to $23 \%, 25 \%$ and $31 \%$ respectively, and would occur in the 2070 period in a pessimistic scenario (RCP 8.5). This is the first study that predicts the potential suitable areas for passiflora's crops, using the MaxEnt model and contemplating climate change scenarios on a regional scale in a tropical region. The proposed approach can provide important tools for the management and sustainable use of the species studied.

Keywords: Passiflora edulis var. flavicarpa Degener, Passiflora ligularis Juss, Passiflora maliformis L., Maxent 


\section{RESUMEN GRÁFICO}

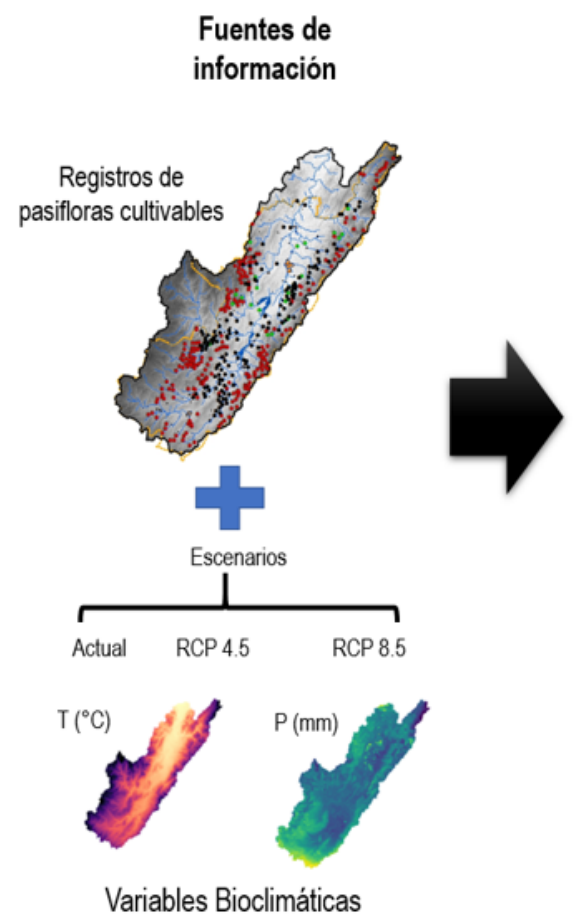

Fuentes de información

Fuente: autores
Modelo de Distribución de Especies MDE

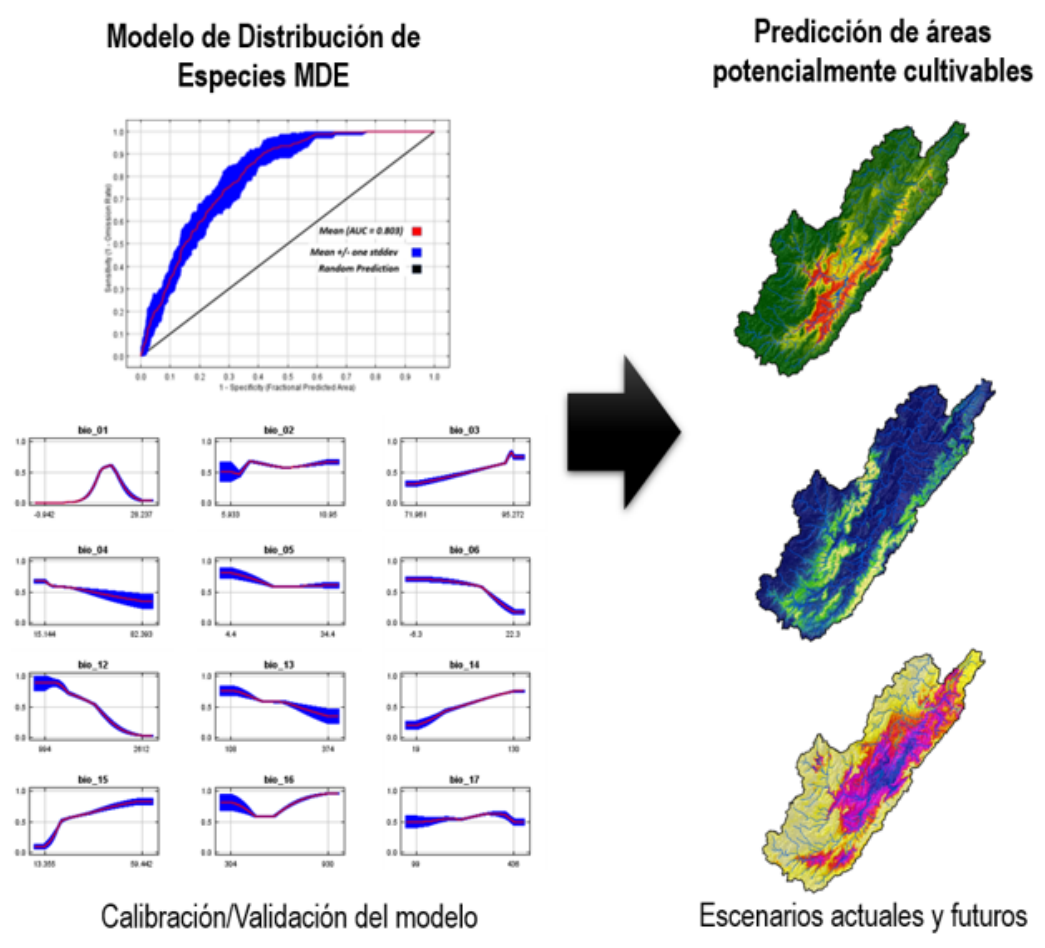

\section{INTRODUCCIÓN}

Uno de los principales desafíos que enfrenta la humanidad hoy en día es el cambio climático, el cual tiene impacto en los ecosistemas, la sociedad y la economía. Los impactos del cambio climático y los cambios en el uso/cobertura del suelo son dos de los principales causantes de la pérdida de biodiversidad, redistribución geográfica de las especies y disminución de la producción agrícola en gran escala (Pecl et al., 2017; Pareek, et al., 2020; Giannini et al., 2013). Sin embargo, los impactos del cambio climático a nivel de ecosistemas y comunidades, así como los efectos en cultivos agrícolas en pequeña escala, son poco estudiados.

En las próximas décadas se tiene previsto que el cambio climático podría impactar drásticamente la distribución de especies, especialmente en los bordes de las ecoregiones, causando fragmentación, acelerando la pérdida de biodiversidad (Werneck et al., 2011; Scanes, 2018), afectando directamente cultivos agrícolas (Lobell et al., 2011, Polce et al., 2014, Rosenzweig et al., 2014) y polinizadores (Bartomeus et al., 2013; Burkle et al., 2013;
Vanbergen \& the Insect Pollinators Initiative, 2013; Elias et al., 2017). Así mismo, los cambios significativos en los patrones de las principales variables meteorológicas (p. ej., temperatura y precipitación) que se prevén para el final del siglo XXI (Intergovernmental Panel on Climate Change [IPCC], 2013a), conllevan a la necesidad de proponer herramientas de gestión que ayuden a la conservación de los ecosistemas frente a diferentes usos, y que permitan el entendimiento de la dinámica, estructura, funcionamiento y monitoreo.

En los últimos años, se han desarrollado modelos de distribución de especies (MDE) para proyectar las pasadas, presentes y futuras condiciones climáticas, así como estudiar la distribución geográfica de diversas especies, lo que permite identificar áreas de estabilidad climática y predecir los impactos del cambio climático sobre la diversidad de especies (Thuiller et al., 2004; Collevatti, et al., 2011; Espíndola et al., 2012; Collevatti et al., 2014). Dentro de los MDE más utilizados se incluyen los modelos GARP (Stockwell, 1999), BIOCLIM (Nix, 1986), CLIMEX (Sutherst \& Maywald, 1985) y Máxima 
Entropía [Maxent] (Phillips et al., 2006), siendo este último, uno de los modelos que presenta más ventajas, entre ellas: (1) Los datos de las especies de entrada pueden ser datos de solo presencia; (2) se puede producir directamente un mapa de ocurrencia de hábitat de la especie analizada; (3) se puede evaluar la importancia de las variables ambientales de manera individual a través de la prueba de Jackknife; (4) se pueden utilizar datos continuos y categóricos como variables de entrada.

Recientes estudios han empleado el modelo MaxEnt y escenarios del CMIP5 (Coupled Model Intercomparison Project Phase 5) (IPCC, 2013b) para investigar el efecto del cambio climático en cultivos agrícolas en gran escala (Khubaib et al, 2021; Läderach et al., 2017; Shukla et al., 2020; Su et al., 2021). Estos estudios han sido de gran interés para determinar la redistribución geográfica de especies agrícolas, especialmente aquellas más vulnerables al cambio climático y a las condiciones ambientales como las pasifloras (Fernandes et al., 2019; Scherer, 2014; Giannini, et al., 2013). Las pasifloras comprenden alrededor de 520 especies (Wohlmuth et al., 2010) que se encuentran predominantemente en regiones tropicales y subtropicales (Araújo et al., 2017) y su distribución geográfica depende directamente de la temperatura y la precipitación (Scherer, 2014).

Colombia posee la mayor diversidad de especies de la familia Passifloraceae en el mundo, registrando 170 especies (Ocampo et al., 2007; Ocampo, 2013a), siendo las más ultivadas: maracuyá (Passiflora edulis var. Flavicarpa Degener), granadilla (Passiflora ligularis Juss), gulupa (Passiflora edulis $\mathrm{f}$. edulis Sims), curuba (Passiflora tripartita var. mollissima) y cholupa (Passiflora maliformis L.) (Fischer \& Miranda, 2021). El país es el tercer mayor productor mundial de maracuyá ( $5 \%)$ por detrás de India ( 9\%) y Brasil ( 60\%) (United States Agency for International Development [USAID], 2014).

Especies cultivables de pasifloras, como el maracuyá, la granadilla y la cholupa, se han visto fuertemente amenazadas por el cambio climático (Bezerra et al., 2019; Loaiza y Mesa, 2019; Kjøhl et al., 2011; Burkle et al., 2013;
Vanbergen y The Insect Pollinators Initiative, 2013; Polce et al., 2014; Setteleet al., 2016; Bejarano y Ibarra, 2018; Montero y Laiton, 2016), lo que impacta la polinización natural y disminuye su rendimiento. Sin embargo, en la escala regional existen muy pocos estudios que determinen las condiciones climáticas futuras y la redistribución geográfica de estas especies cultivables, por medio de modelos de distribución, contemplando escenarios de cambio climático. Teniendo en cuenta esto, el objetivo de este estudio fue estimar las potenciales áreas cultivables actuales y futuras de maracuyá (Passiflora edulis var. flavicarpa Degener), granadilla (Passiflora ligularis Juss) y cholupa (Passiflora maliformis L.) en una región tropical, usando el modelo MaxEnt, considerando escenarios de cambio climático (Representative Concentration Pathway [RCP 4.5 y RCP 8.5].

\section{MATERIALES Y MÉTODOS}

\section{1 Área de estudio}

El área seleccionada para el estudio fue la cuenca alta del río Magdalena $\left(1^{\circ} 29^{\prime}\right.$ y $3^{\circ} 52^{\prime} \mathrm{N}, 74^{\circ} 23^{\prime}$ y $76^{\circ} 39^{\prime} \mathrm{W}$ ), Colombia (Fig. 1), que abarca el departamento del Huila. La selección de la cuenca se debió a que el departamento del Huila es el responsable del $45 \%$ de la producción nacional de maracuyá, del 50 \% de la producción de granadilla en el país y además lidera la producción nacional de cholupa (Ministerio de Agricultura y Desarrollo Rural [Minagricultura], 2018). Para la delimitación de la cuenca se utilizó el modelo digital de elevación MDE derivado de la misión SRTM (Shuttle Radar Topography Mission) (Farr et al., 2007), que cuenta con una resolución espacial de $90 \mathrm{~m}$ (United States Geological Survey [USGS], 2005).

A partir del MDE se realizaron operaciones de geoprocesamiento utilizando el software ArcGIS 10.2. La secuencia de operaciones fue la siguiente:

- Relleno de fallas del modelo digital de elevación, cuyo objetivo fue corregir valores nulos en la matriz y zonas muertas de flujo.

- Determinación de la dirección de flujo para estimar el camino más probable 


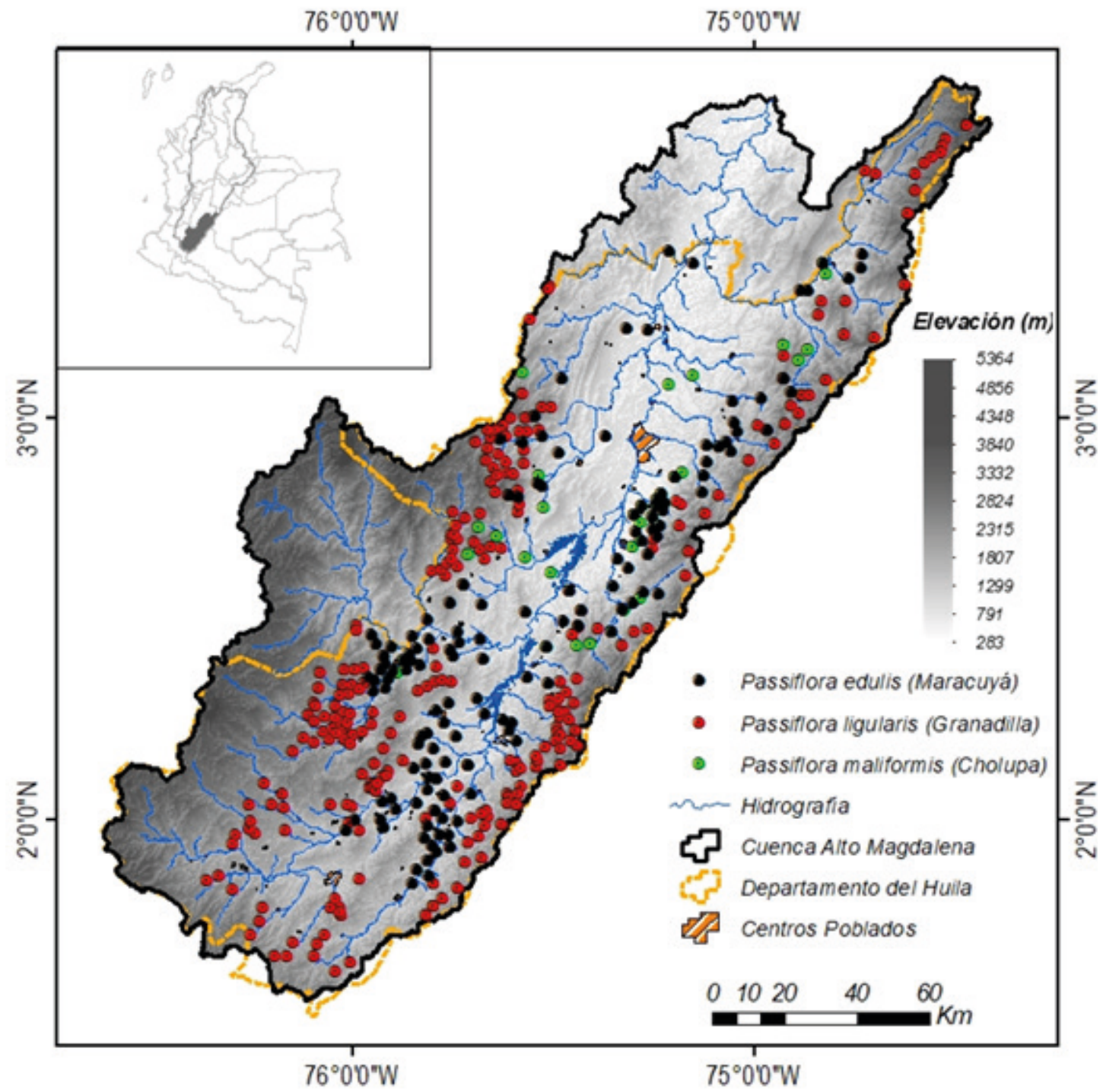

Figura 1. Distribución espacial de registros de presencia de cultivos de maracuyá, granadilla y cholupa en la cuenca alta del río Magdalena.

Fuente: autores.

para la red de drenaje a partir de una evaluación de declividad de cada píxel.

- Mapeo del flujo acumulado para determinar el área drenada por cada píxel.

- Definición del área de drenaje de la cuenca.

-Definición de la red de drenaje.

La cuenca alta del río Magdalena presenta un relieve variado, con altitudes máximas de 5364 m.s.n.m y mínimas de 283 m.s.n.m. En la cuenca alta, nace el río Magdalena, el mayor sistema fluvial de Colombia (área aproximada $257.000 \mathrm{~km}^{2}$ ). Así mismo, en la cuenca del rio Magdalena se encuentran las principales ciudades del país (Bogotá, Cali, Medellín, Barranquilla) y se concentra aproximadamente el $80 \%$ de la población colombiana (Restrepo \& Syvitski, 2006).

\subsection{Datos bióticos}

Los registros de presencia de cultivos de maracuyá, granadilla y cholupa, y su distribución espacial a partir de sus coordenadas geográficas, se obtuvieron de la base de datos de la Evaluación Agropecuaria del Huila (Gobernación del Huila, 2020). En total, se analizaron 141 registros de presencia de maracuyá, 256 registros de granadilla y 40 registros de cholupa distribuidos en la 
cuenca alta del río Magdalena (Fig. 1). Todos los registros de presencia se importaron a Microsoft Excel y se guardaron en formato "CSV" para utilizarse como datos de entrada para el modelo de distribución de especies MDE.

\subsection{Datos bioclimáticos}

Los datos de variables bioclimáticas, utilizadas como entrada para el modelo MaxEnt, se obtuvieron en resolución de 30 segundos $\left(\sim 1 \mathrm{~km}^{2}\right)$ desde la plataforma WorldClim (Version 2.0, ) para el período actual (Fick \& Hijmans, 2017). En la base de datos de Worldclim, el "período actual" se definió entre los años 1970 a 2000 (estos datos se han utilizado ampliamente en la creación de modelos de distribución de especies). En total, se utilizaron 19 variables bioclimáticas de temperatura y precipitación para este período. Es de anotar que las variables bioclimáticas se derivan de los valores mensuales de temperatura y precipitación para generar variables más significativas desde el punto de vista ecológico. Estas variables representan tendencias anuales ( $p$. ej., temperatura media anual, precipitación anual), estacionalidad (p. ej., rango anual de temperatura y precipitación) y factores ambientales extremos o limitantes (p. ej., temperatura y precipitación del mes más frio y del más cálido).

Las proyecciones para las condiciones climáticas futuras se obtuvieron con base en los cambios climáticos previstos para los períodos 2050 (average for 2041-2060) y 2070 (average for 2061-2080), teniendo en cuenta los escenarios RCP 4.5 y RCP 8.5 (Representative Concentration Pathway) desarrollados por Hadley Center Global Environmental Model (HadGEM2-ES) (Collins et al., 2011) y siguiendo la metodología sugerida por Bezerra et al. (2019). Según el Quinto Informe de Evaluación del IPCC (AR5) (IPCC, 2013b), cada escenario RCP representa un "Camino Representativo de Concentración" de Gases Efecto Invernadero [GEI] y de aerosoles en la atmósfera. Los RCP se describen por su Forzamiento Radiactivo (FR) total para el año 2100 , siendo 4.5 y 8.5 vatios por metro cuadrado $\left(\mathrm{W} / \mathrm{m}^{2}\right)$ los escenarios analizados en este estudio y que corresponden a los escenarios moderado y pesimista, respectivamente.

\subsection{Modelo de distribución de especies [MDE]}

En esta investigación se utilizó el modelo MaxEnt (Phillips et al., 2006) obtenido de manera gratuita desde http://www. cs.princeton.edu/. Este modelo determina patrones de distribución para los datos ingresados y luego selecciona los de configuración más probable basado en la maximización de entropía de Shannon (Merow et al., 2013, Phillips et al., 2006). El modelo MaxEnt utiliza el principio de máxima entropía para estimar, a partir de los registros de presencia de las especies analizadas y presentes en las capas bioclimáticas, una distribución de probabilidad (variando entre 0 y $100 \%$ ) que puede denominarse como índice de ocurrencia de la especie que se está modelando (Phillips et al., 2006; Phillips et al., 2017).

\subsection{Análisis estadístico y medidas de desempeño}

Para seleccionar las variables bioclimáticas más representativas se realizó un análisis de componentes principales (ACP), considerando como criterios de selección las variables de mayor correlación $\left(R^{2} \geq 0.75\right)$ y el porcentaje de varianza explicado por autovalores ( $>5$ $\%$ ) (ver Anexo 1). El ACP fue realizado en el software ArCGIS 10.2, siguiendo la metodología propuesta por Moya et al., (2017). A partir de las 19 variables bioclimáticas de temperatura y precipitación analizadas, se seleccionaron 12 variables bioclimáticas de acuerdo con los resultados del ACP (Tabla 1, Anexo 1). Las variables de menor contribución se excluyeron del modelo final para evitar la multicolinealidad de los modelos.

El $75 \%$ de los registros de presencia de cada especie, seleccionados al azar, se utilizaron para la etapa de calibración del modelo y el $25 \%$ restante se utilizó para la etapa de validación. Para evaluar el rendimiento del modelo se calcularon las curvas de disponibilidad de hábitat de cada variable y se calcularon las contribuciones de cada una, utilizando la prueba de Jackknife. Esta prueba se empleó para medir los factores climáticos dominantes que determinan la distribución potencial de las especies, identificando así las variables bioclimáticas con la mayor contribución (Moratelli et al., 2011).

Para evaluar el desempeño del modelo MaxEnt se utilizaron la curva ROC (Receiver 
Tabla 1. Variables bioclimáticas seleccionadas como datos de entrada para el modelo Maxent de acuerdo con el ACP.

Código de la variable bioclimática

\section{Descripción}

Temperatura media anual

BIO_02

Rango diurno medio de temperatura

BIO_03

Isotermalidad (BIO2 / BIO7) (* 100)

BIO_04

Estacionalidad de temperatura (desviación estándar * 100)

BIO_05

Temperatura máxima del mes más cálido

BIO_06

Temperatura mínima del mes más frío

BIO_12

Precipitación anual

BIO_13

Precipitación del mes más húmedo

BIO_14

Precipitación del mes más seco

BIO_15

BIO_16

Estacionalidad de precipitación (Coeficiente de variación)

BIO_17

Precipitación del trimestre más seco

Fuente: autores

Operating Characteristic) y el área bajo la curva ROC (Area Under Curve [AUC]), a través de la plataforma Niche Toolbox (Nichetoolbox, 2016; Peterson et al., 2008), lo que permite evaluar las predicciones del modelo que presentan errores de omisión inferiores al $10 \%$. El desempeño del modelo se clasificó según los valores de AUC, siendo fallido ( $0.5 \leq$ AUC $<0.6)$, pobre $(0.6 \leq$ AUC $<0.7)$, razonable $(0.7 \leq \mathrm{AUC}<0.8)$, bueno $(0.8 \leq$ AUC $<0.9)$ y excelente $(0.9 \leq$ AUC $\leq 1.0$ ) (Swets, 1988).

A partir del modelo MaxEnt calibrado/ validado se proyectaron las distribuciones actuales y futuras para los períodos 2050 y 2070 en los escenarios RCP 4.5 y RCP 8.5 (Fig. 2). Las distribuciones fueron basadas en el promedio logístico de 10 réplicas siguiendo la metodología sugerida por Wei et al. (2018), para estimar los índices de ocurrencias que varían entre $0 \%$ (no es probable que ocurra) y $100 \%$ (es más probable que ocurra) (Phillips et al., 2006).

\section{RESULTADOS Y DISCUSIÓN}

\subsection{Desempeño del modelo contribución e importancia variables bioclimáticas \\ MaxEnt: de las}

Los resultados revelan una buena predicción del modelo MaxEnt para las especies de pasifloras: maracuyá, granadilla y cholupa, en la cuenca alta del río Magdalena, con valores de AUC de 0.844; 0.803 y 0.742 respectivamente (Fig. 3).

Estos resultados fueron consistentes con estudios similares aplicando el modelo Maxent para predecir la distribución potencial de maracuyá (Bezerra et al, 2019; Giannini et al., 2013; Elias et al., 2017; Ocampo et al., 2013) y especies forestales (Wei at al., 2018; Yuan et al., 2015; Remya et al., 2015; Yi et al., 2016; Sharma et al., 2018). Para el cultivo de cholupa, los resultados fueron razonables $0.7<A U C<0.8)$. Este comportamiento puede ser debido al número bajo de registros de presencia de la especie (40 registros) utilizados para la calibración del modelo MaxEnt y a las variables bioclimáticas empleadas, que pueden superar del rango de registros observados de la especie (Kumar, 2012).

Las variables bioclimáticas seleccionadas a partir del ACP describieron bien la distribución actual de maracuyá, granadilla $(0.8 \leq A U C<0.9)$ y, de manera razonable, la distribución de cholupa (0.7 $\leq$ AUC <0.8). Entre las 12 variables bioclimáticas (Fig. 4), las variables que más contribuyen en los pronósticos de las especies analizadas fueron BIO_05 (temperatura máxima del mes más cálido y BIO_01 (temperatura media anual), mientras que las variables de mayor 


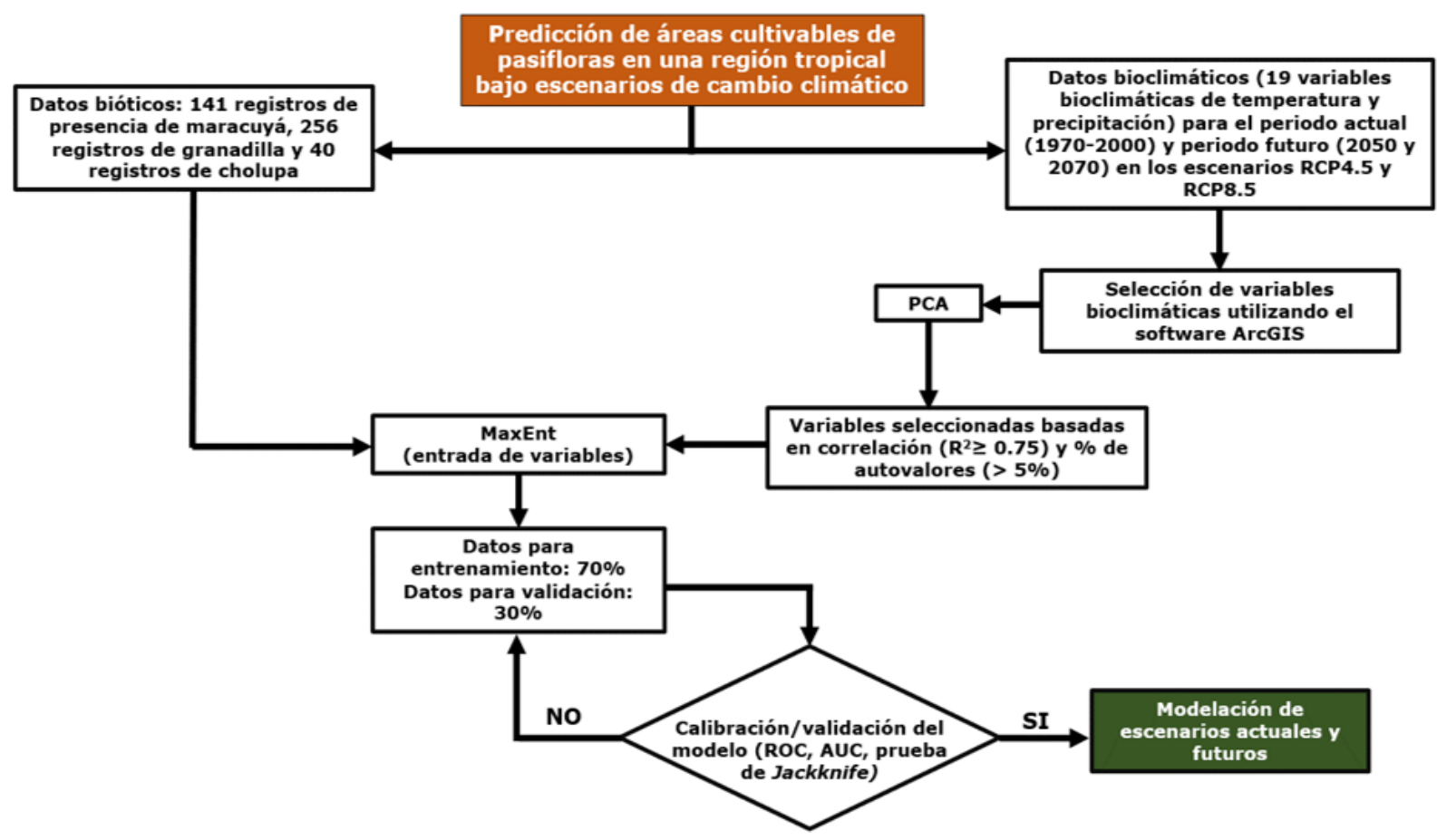

Figura 2. Diagrama de flujo para la predicción de áreas cultivables de pasifloras en una región tropical utilizando el modelo MaxEnt considerando escenarios de cambio climático.

Fuente: autores.

a)

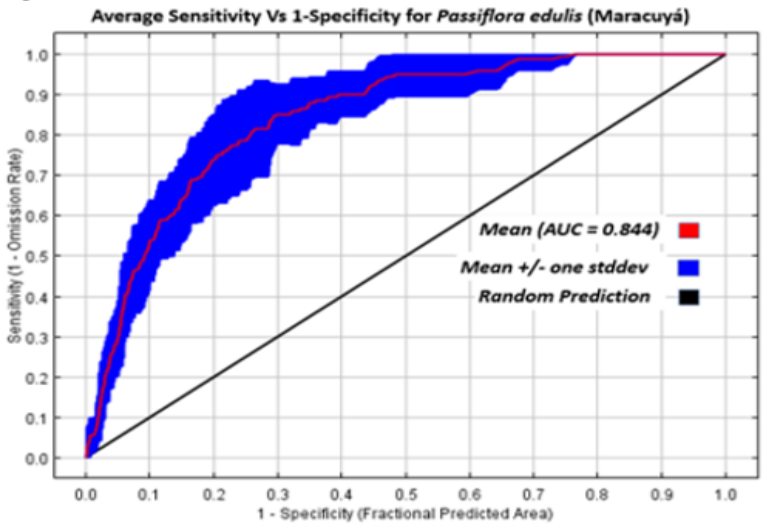

b)

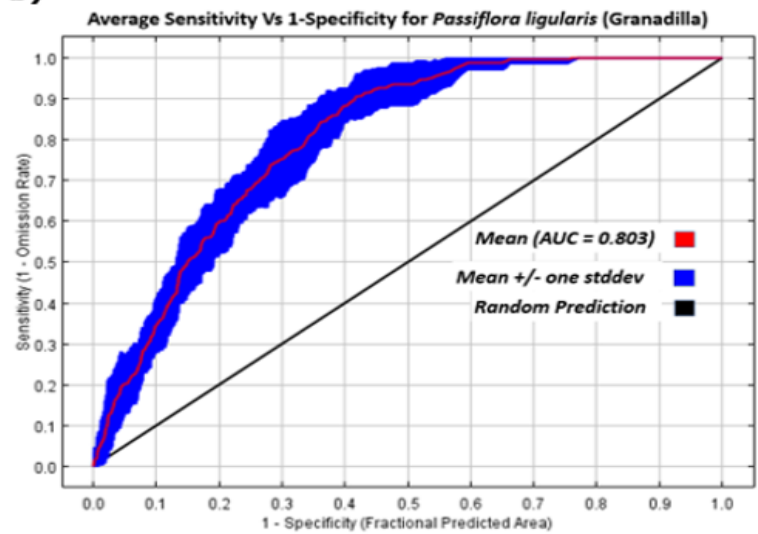

c)

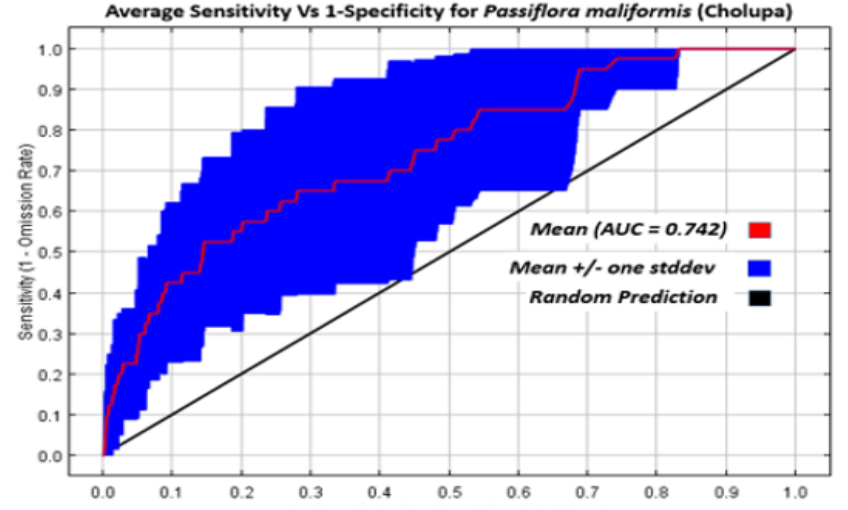

Figura 3. Curvas ROC y valores de AUC para el período actual (10 réplicas) para a) maracuyá, b) granadilla, y c) cholupa.

Fuente: autores 
importancia en la permutación corresponden a BIO_01 (Temperatura media anual) y BIO_04 (Estacionalidad de temperatura (desviación estándar * 100)). Dentro de las variables de precipitación, la variable BIO_14 (Precipitación del mes más seco) es la que más tiene importancia en la permutación

a)

Passiflora edulis (Maracuyá)

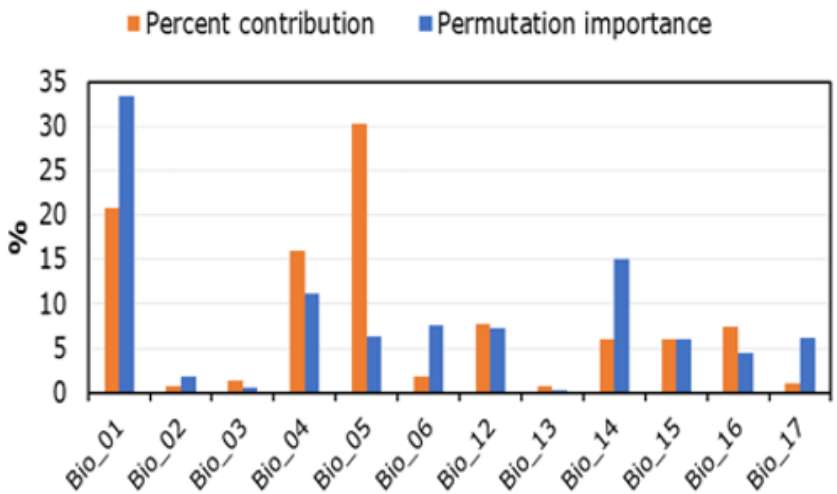

c)

\section{Passiflora maliformis (Cholupa)}

= Percent contribution $=$ Permutation importance

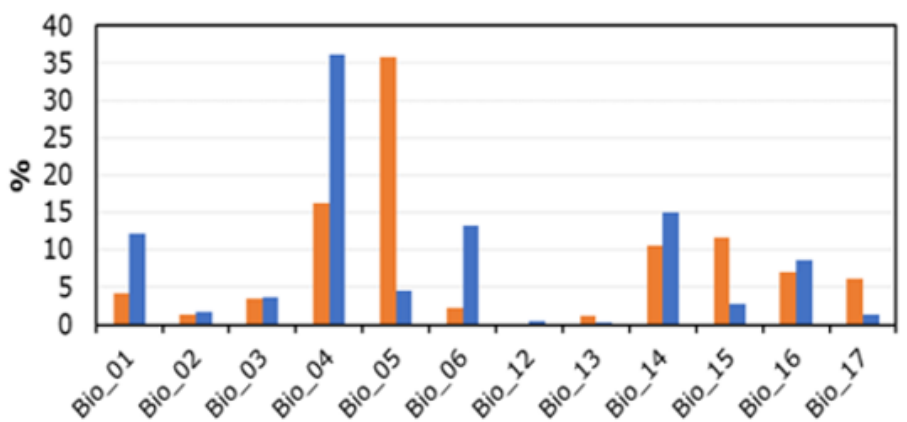

Figura 4. Contribución e importancia de permutación de cada variable analizada en el período actual (10 réplicas) para a) maracuyá, b) granadilla y c) cholupa.

Fuente: autores.

Los resultados de la prueba de Jacknife (Fig. 5) corroboran que las variables BIO_01 y BIO_05 son las de mayor contribución e importancia de permutación. Sin embargo, la variable bioclimática BIO_06 (Temperatura mínima del mes más frío) también reveló significativamente alta ganancia y contribución en los resultados obtenidos para los pronósticos de la distribución actual de las especies analizadas, en comparación con las otras variables utilizadas. Esta variable es importante, debido a que las especies analizadas son altamente sensibles a las heladas y esto puede causar daños severos con relación a las otras variables analizadas. Este comportamiento puede darse debido a que las especies analizadas son sensibles a los cambios drásticos de temperatura y precipitación, lo que puede afectar su crecimiento, desarrollo y producción (Fischer \& Miranda, 2021; Melgarejo, 2015).

b)

\section{Passiflora ligularis (Granadilla)}

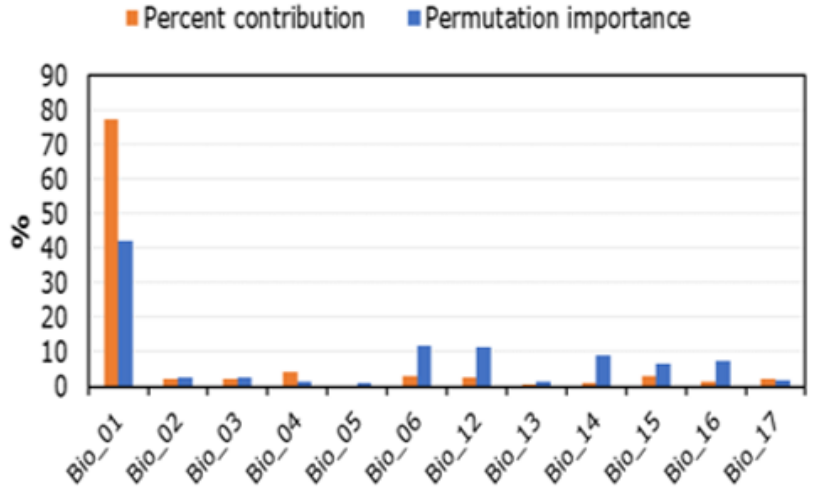


Jackknife of regularized training gain for Passiflora edulis (Maracuyá)

Without variable $=$ With only variable $\|$ With all variables $=$

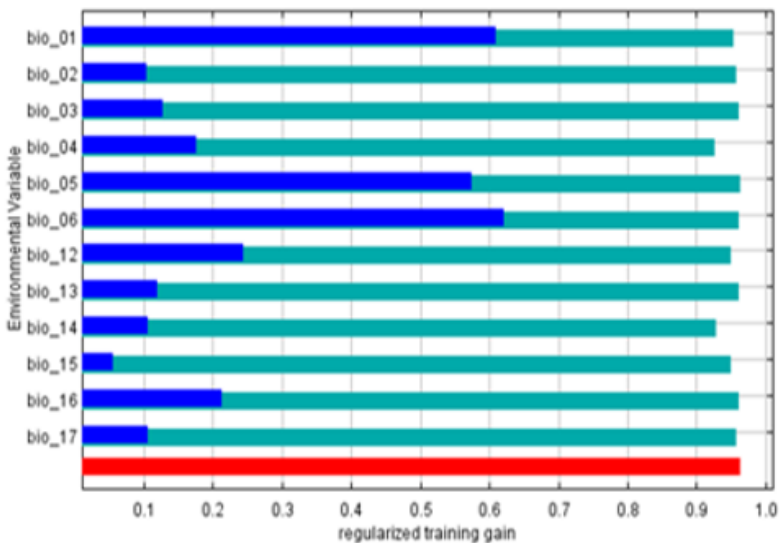

Jackknife of regularized training gain for Passiflora ligularis (Granadilla)

Without variable $=$ With only variable $=$ With all variables $=$

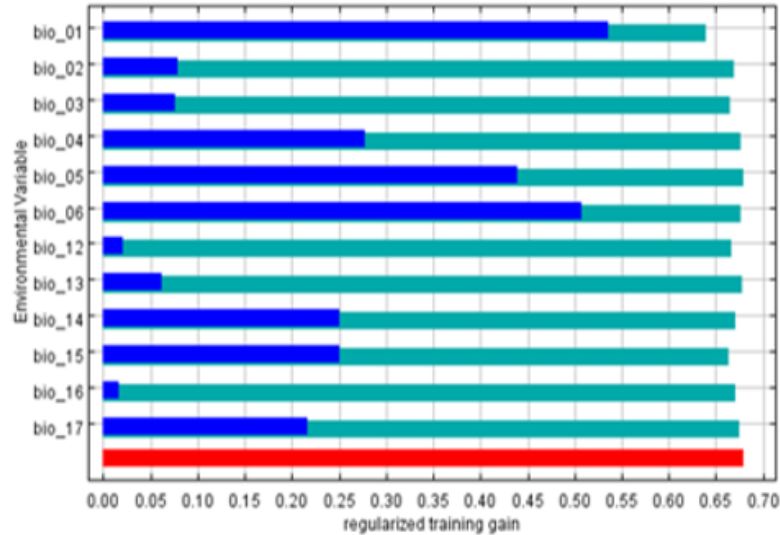

c)

Jackknife of regularized training gain for Passiflora maliformis

(Cholupa)

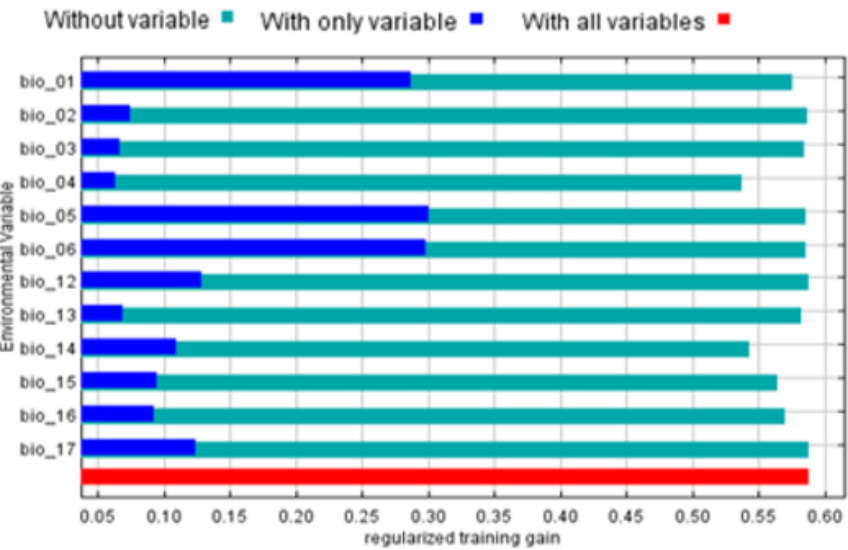

Figura 5. Test de Jackknife de la importancia de cada variable en el período actual (10 réplicas) para a) maracuyá, b) granadilla y c) cholupa.

Fuente: autores.

Tabla 2. Desempeño del modelo MaxEnt en la distribución de maracuyá, granadilla y cholupa, a partir de dos escenarios diferentes de RCP (4.5 y 8.5) en dos períodos futuros (2050 y 2070 ).

\begin{tabular}{|c|c|c|c|c|}
\hline Especie & \multicolumn{2}{|c|}{ Período } & AUC promedio & AUC promedio desviación estándar \\
\hline \multirow{4}{*}{ Maracuyá } & 2050 & RCP 4.5 & 0.850 & 0.034 \\
\hline & & RCP 8.5 & 0.855 & 0.033 \\
\hline & 2070 & RCP 4.5 & 0.852 & 0.035 \\
\hline & & RCP 8.5 & 0.855 & 0.042 \\
\hline \multirow[t]{4}{*}{ Granadilla } & 2050 & RCP 4.5 & 0.815 & 0.024 \\
\hline & & RCP 8.5 & 0.818 & 0.019 \\
\hline & 2070 & RCP 4.5 & 0.817 & 0.016 \\
\hline & & RCP 8.5 & 0.815 & 0.042 \\
\hline \multirow[t]{4}{*}{ Cholupa } & 2050 & RCP 4.5 & 0.814 & 0.044 \\
\hline & & RCP 8.5 & 0.821 & 0.059 \\
\hline & 2070 & RCP 4.5 & 0.798 & 0.049 \\
\hline & & RCP 8.5 & 0.745 & 0.085 \\
\hline
\end{tabular}

Fuente: autores 


\subsection{Modelación de escenarios actuales y futuros con MaxEnt}

La distribución potencial en los escenarios actuales y futuros de las potenciales áreas cultivables de maracuyá, granadilla y cholupa en la cuenca alta del río Magdalena se realizó considerando las salidas de la modelación con MaxEnt, con el apoyo del software ArcGIS 10.2. La estimación de pérdida de áreas de ocurrencia se hizo comparando el escenario actual y los escenarios moderado (RCP 4.5) y pesimista (RCP 8.5) para las 3 especies analizadas.

Para el caso del cultivo de maracuyá (Fig. $6)$, los pronósticos del modelo revelan una pérdida de las áreas de ocurrencia (mayor en la parte baja de la cuenca), especialmente en las regiones comprendidas entre los 400 y 1.100 m. s. n. m., donde el porcentaje de ocurrencia esperado de la especie es bajo. Por otro lado, los resultados revelan áreas con mayor porcentaje de ocurrencia (> 60 $\%)$ de la especie en la parte alta de la cuenca en los escenarios futuros, donde se espera una redistribución geográfica de la especie en zonas con altitudes comprendidas entre 1.100 y 1.600 m. s. n. m. Los resultados revelan que para el período 2070, en el escenario pesimista (RCP 8.5), se pronostican las mayores pérdidas en las áreas de ocurrencia con valores superiores al $23 \%$ (Tabla 3), mientras que en el escenario moderado (RCP 4.5) se podrían presentar pérdidas en las áreas de ocurrencia con valores máximos del $6.3 \%$ y mínimos del $4.95 \%$. Estos resultados se han observado previamente en ambientes similares (Bezerra et al, 2019; Giannini et al., 2013), donde se evidencia una disminución en las áreas de ocurrencia de la especie y de sus polinizadores debido al cambio climático. Así mismo, a partir de los resultados obtenidos, se observa una redistribución de la especie a zonas potenciales de mayor altitud, principalmente debidas al aumento en la temperatura, disminución en la precipitación (menor disponibilidad de agua) y nuevas características fisicoquímicas del suelo. En estas nuevas zonas de redistribución será necesario la implementación de nuevas prácticas agronómicas, debido a los posibles cambios en la fisiología de la especie (Ocampo et al., 2013).

Con relación al cultivo de granadilla (Fig. 7, Tabla 3), los resultados obtenidos indican pérdidas en las áreas de ocurrencia ligeramente mayores a las pronosticadas en el cultivo de maracuyá. La redistribución geográfica de las áreas potenciales para el cultivo presenta un porcentaje mayor de ocurrencia ( $>70 \%$ ) en zonas con altitudes comprendidas entre 1300 y 2300 m. s. n. m., coincidiendo con lo reportado previamente por Osorio et al. (2020). Así mismo, los resultados revelan que las pérdidas esperadas en las áreas de ocurrencia para el período 2070 son del orden de $4.95 \%$ para el escenario moderado (RCP 4.5) y cercanas al $25 \%$ en el escenario pesimista (RCP 8.5).

Los resultados obtenidos para el cultivo de cholupa revelan la mayor pérdida en las áreas de ocurrencia pronosticadas por MaxEnt, en comparación con las demás especies analizadas (Fig. 8, Tabla 3). El mayor porcentaje de pérdida en las áreas de ocurrencia se pronostica para las zonas con altitudes bajas comprendidas entre 400 y 1000 m. s. n. m. Los pronósticos muestran que las mayores pérdidas en las áreas de ocurrencia en el escenario pesimista (RCP 8.5 ) son superiores al $31 \%$ para el período 2070, mientras que las menores pérdidas se pronostican en el escenario moderado (RCP 4.5) para el período 2050 con pérdidas del orden del $6 \%$ en las áreas de ocurrencia. Sin embargo, la información existente sobre la especie es limitada, siendo en la mayoría de los casos extrapolada de información proveniente del cultivo de maracuyá (Ocampo et al., 2015). 

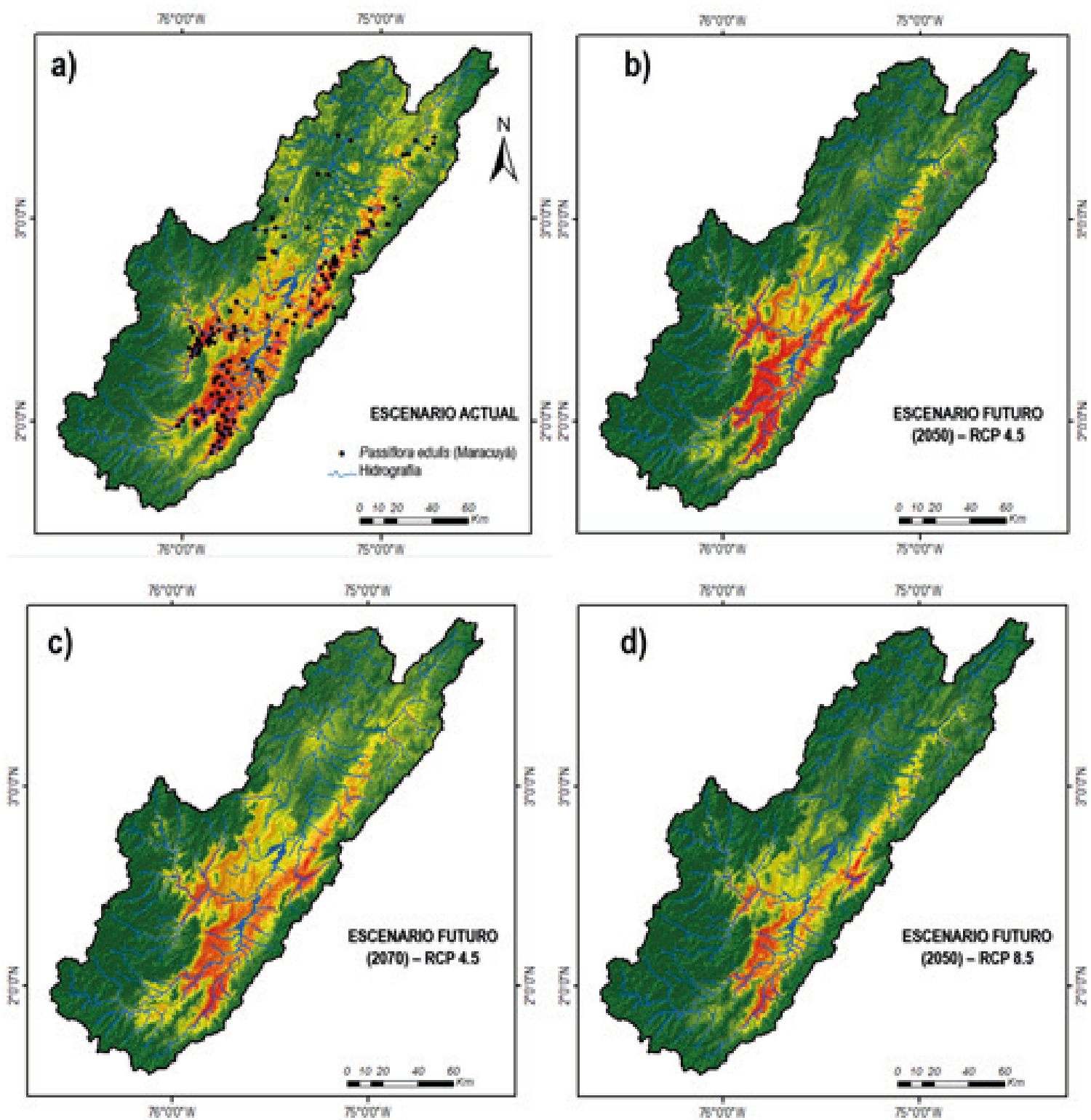

Figura 6. Áreas de distribución del cultivo de maracuyá en la cuenca alta del río Magdalena para el periodo actual (1970 a 2000) y dos períodos futuros (2050 y 2070) contemplando escenarios diferentes de RCP (4.5 y 8.5).

Fuente: autores

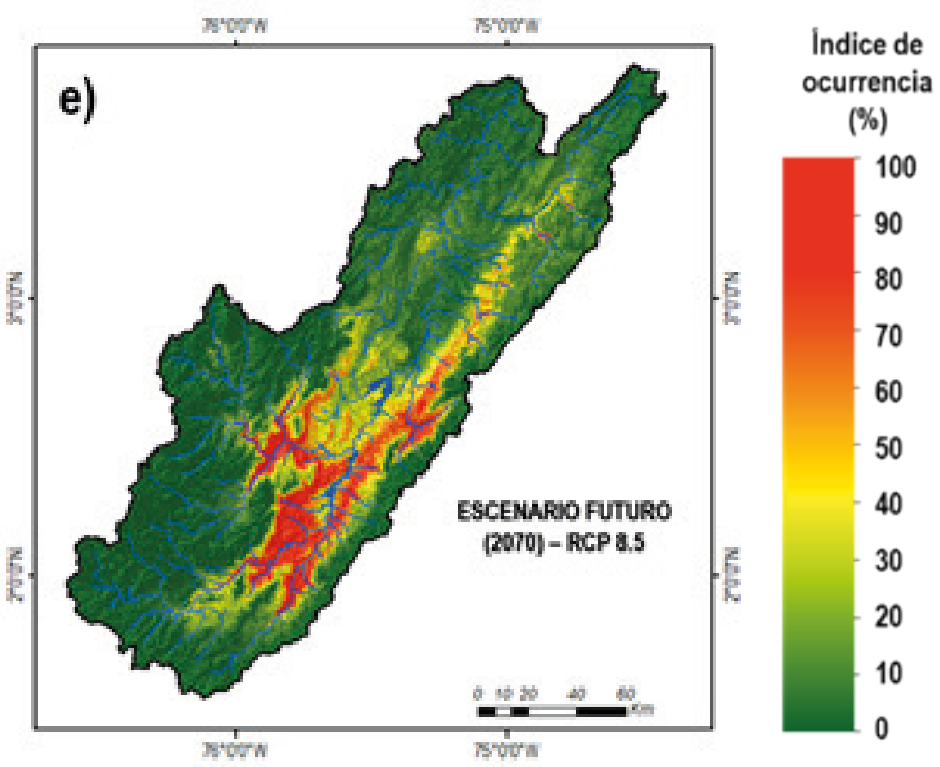



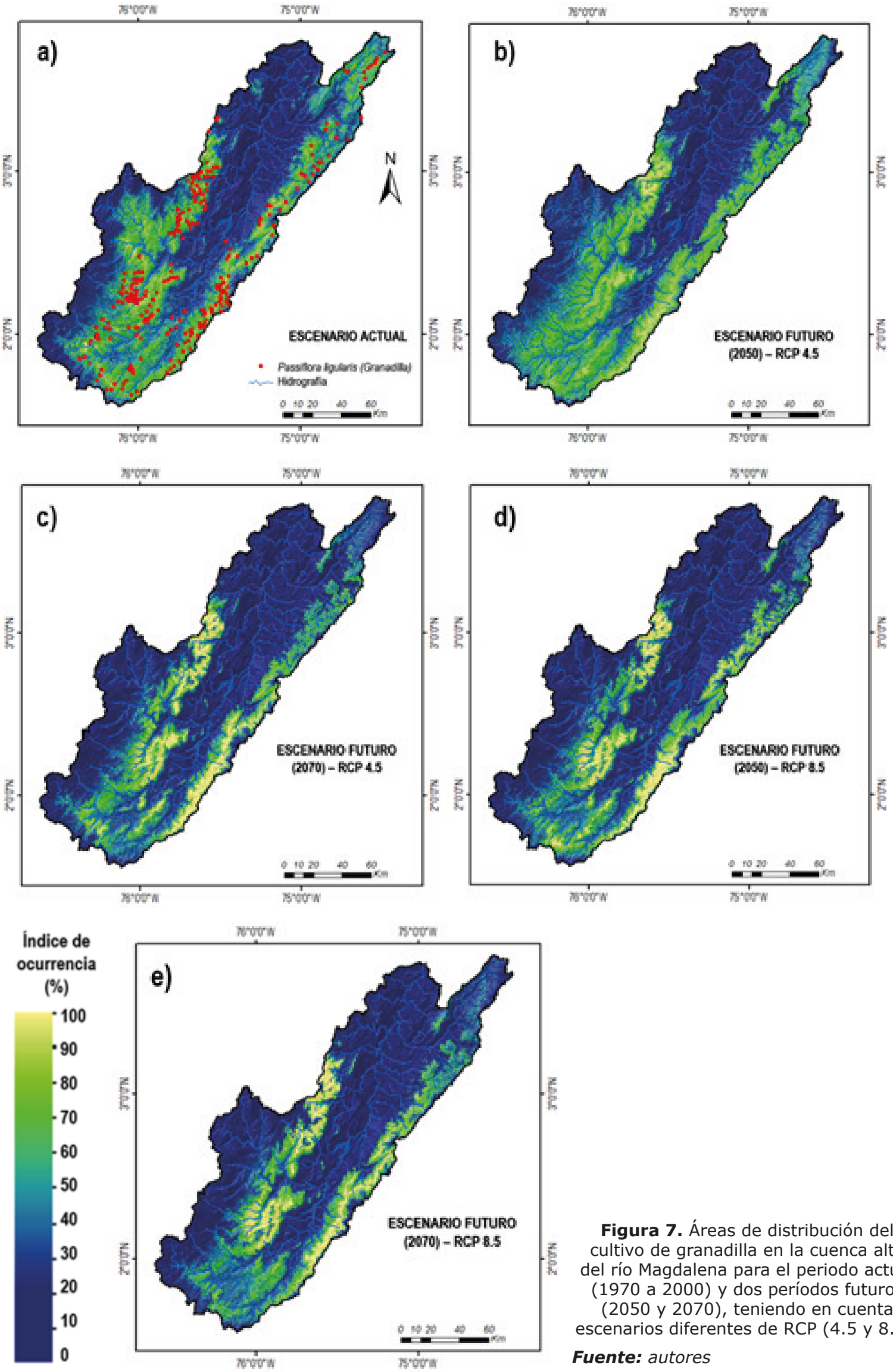

Figura 7. Áreas de distribución del cultivo de granadilla en la cuenca alta del río Magdalena para el periodo actual

(1970 a 2000) y dos períodos futuros

(2050 y 2070), teniendo en cuenta escenarios diferentes de RCP (4.5 y 8.5).

Fuente: autores 

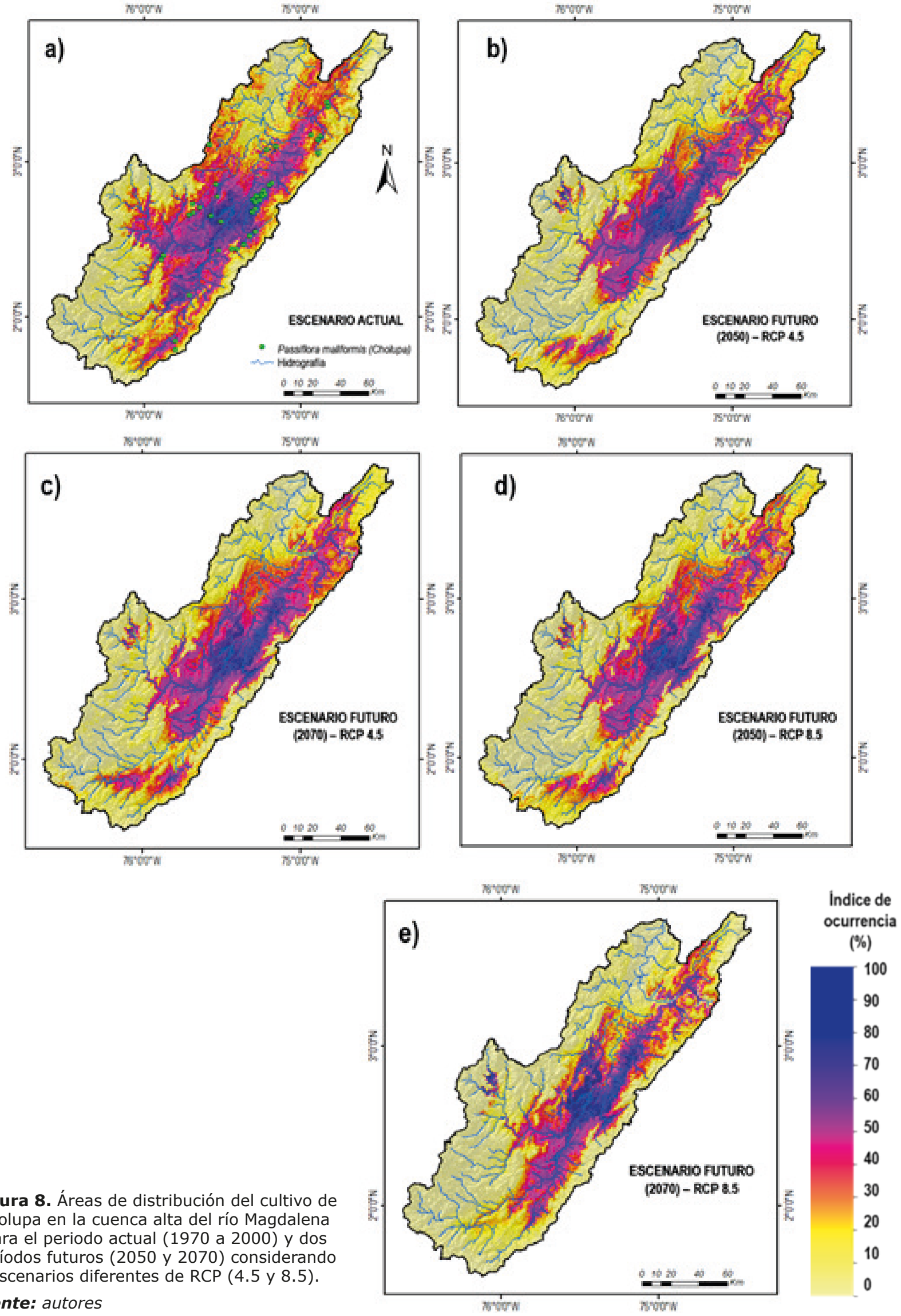

Figura 8. Áreas de distribución del cultivo de cholupa en la cuenca alta del río Magdalena para el periodo actual (1970 a 2000) y dos períodos futuros (2050 y 2070) considerando escenarios diferentes de RCP (4.5 y 8.5).

Fuente: autores 
En la siguiente tabla se presenta el resumen de los pronósticos del modelo MaxEnt con la estimación de las áreas de ocurrencia potencial, pérdidas de áreas estimadas en el escenario actual y pérdidas de área estimadas en futuros escenarios (RCP $4.5,2050 ; 2070$ y RCP $8.5,2050 ; 2070$ ) para los cultivos de maracuyá, granadilla y cholupa en la cuenca alta del río Magdalena.

Tabla 3. Áreas de ocurrencia potencial y pérdidas de áreas en el escenario actual y los futuros escenarios (RCP $4.5,2050 ; 2070$ y RCP $8.5,2050 ; 2070$ ) para los cultivos de maracuyá, granadilla y cholupa en la cuenca alta del río Magdalena.

\begin{tabular}{|c|c|c|c|c|}
\hline Especie & Escenario & $\begin{array}{c}\text { Areas de } \\
\text { ocurrencia } \\
\text { potencial } \\
\text { (ha) }\end{array}$ & $\begin{array}{l}\text { Potencial } \\
\text { (ha) }\end{array}$ & $\begin{array}{c}\text { Pérdida de área } \\
\qquad(\%)\end{array}$ \\
\hline \multirow{4}{*}{ Maracuyá } & Actual Vs RCP 4.5 (2050) & $64.242,00$ & $3.182,00$ & $4,95 \%$ \\
\hline & Actual Vs RCP 4.5 (2070) & $64.242,00$ & $4.042,00$ & $6,29 \%$ \\
\hline & Actual Vs RCP 8.5 (2050) & $64.242,00$ & $4.902,00$ & $7,63 \%$ \\
\hline & Actual Vs RCP 8.5 (2070) & $64.242,00$ & $15.050,00$ & $23,43 \%$ \\
\hline \multirow{4}{*}{ Granadilla } & Actual Vs RCP 4.5 (2050) & $52.116,00$ & $1.548,00$ & $2,97 \%$ \\
\hline & Actual Vs RCP 4.5 (2070) & $52.116,00$ & $2.580,00$ & $4,95 \%$ \\
\hline & Actual Vs RCP 8.5 (2050) & $52.116,00$ & $3.956,00$ & $7,59 \%$ \\
\hline & Actual Vs RCP 8.5 (2070) & $52.116,00$ & $12.986,00$ & $24,92 \%$ \\
\hline \multirow{4}{*}{ Cholupa } & Actual Vs RCP 4.5 (2050) & $44.376,00$ & $2.666,00$ & $6,01 \%$ \\
\hline & Actual Vs RCP 4.5 (2070) & $44.376,00$ & $5.246,00$ & $11,82 \%$ \\
\hline & Actual Vs RCP 8.5 (2050) & $44.376,00$ & $6.966,00$ & $15,70 \%$ \\
\hline & Actual Vs RCP 8.5 (2070) & $44.376,00$ & $13.846,00$ & $31,20 \%$ \\
\hline
\end{tabular}

Fuente: autores

Las potenciales áreas de ocurrencia cultivables para las 3 especies analizadas sugieren que las especies pueden cambiar su actual área de ocurrencia hacia nuevas áreas, especialmente aquellas localizadas en la parta alta de la cuenca del río Magdalena. Las predicciones a partir del modelo MaxEnt indican que las pérdidas en las áreas de ocurrencia potencial tendrían un aumento gradual (2070> 2050> período actual), siendo mayores en el escenario pesimista (RCP 8.5) que en el moderado (RCP 4.5), como se ha encontrado en previos estudios Donoghue \& Edwards, 2014; Petitpierre et al., 2016). La reducción en las áreas de ocurrencia de las especies analizadas podría generar que la polinización resulte afectada, así como la productividad de los cultivos y, por consiguiente, la economía de las regiones (Settele et al., 2016). Así mismo, los impactos del cambio climático en las áreas de ocurrencia pueden afectar directamente la fenología de algunas especies de plantas y las interacciones planta-polinizador, que se volverían más vulnerables en paisajes modificados (Kjøhl, et al., 2011; Burkle et al., 2013; Vanbergen \& the Insect Pollinators Initiative, 2013; Polce et al., 2014, Settele et al., 2016).

\subsection{Capacidades del modelo MaxEnt y limitaciones}

Los resultados obtenidos a partir del modelo MaxEnt, para la predicción de potenciales áreas cultivables de maracuyá, granadilla y cholupa en la cuenca alta del río Magdalena, muestran que el desempeño del modelo es satisfactorio para representar los patrones de distribución actual y potencial de las especies analizadas considerando escenarios de cambio climático. Los resultados obtenidos pueden ser utilizados por gestores y diseñadores de políticas para la selección del tipo de estrategia que debería implementarse para enfrentar los desafíos del cambio climático. 
Dentro de las limitaciones o incertezas en los resultados obtenidos pueden incluirse:

i. En la modelación con MaxEnt se utilizaron variables bioclimáticas (temperatura y precipitación) excluyendo factores abióticos (p.ej., tipo de suelo, hidrogeología, altitud), que pueden ser variables significativas para la distribución de especies (Zhang et al., 2018). Los factores abióticos no se consideraron debido a que la altitud es una variable proxy que podría generar correlaciones con las variables de temperatura y precipitación, las cuales son suficientes para determinar los límites espaciales de tolerancia climática de las especies
(Kogo et al., 2019; Sun et al., 2013; Woodward, 1987).

ii. Baja resolución espacial de los datos bioclimáticos derivados de la plataforma Worldclim con relación a la cuenca alta del río Magdalena, lo que podría afectar el desempeño del modelo MaxEnt (Kumar, 2012).

iii. Los patrones de distribución actual y potencial derivados de Maxent para las 3 especies analizadas se obtuvieron mediante la extrapolación del modelo, es decir, el modelo MaxEnt se utilizó para hacer predicciones a partir de las variables bioclimáticas que pueden superar el rango de los registros utilizados para calibrar el modelo.

\section{CONCLUSIONES}

Este estudio consistió en la predicción de potenciales áreas cultivables de maracuyá (Passiflora edulis var. flavicarpa Degener), granadilla (Passiflora ligularis Juss), y cholupa (Passiflora maliformis L.), en una región tropical a través del modelo MaxEnt, considerando escenarios de cambio climático. Los resultados revelan que las áreas de ocurrencia potencial para las especies estudiadas disminuirían gradualmente en el futuro, teniendo en cuenta los escenarios climáticos (RCP 4.5 y $R C P \quad 8.5)$ para los períodos 2050 y 2070. Las mayores reducciones en las áreas de ocurrencia potencial se presentarían en el período 2070 para el escenario pesimista (RCP 8.5), con reducciones superiores al $23 \%$ para el cultivo de maracuyá, $25 \%$ para el cultivo de granadilla y superiores al $31 \%$ para el cultivo de cholupa. Los potenciales impactos del cambio climático podrían afectar la fisiología de las especies analizadas siendo necesario de la implementación de nuevas prácticas agronómicas.

Este es el primer estudio que contempla el pronóstico de áreas potencialmente cultivables utilizando el modelo Maxent y escenarios de cambio climático en escala regional en una región tropical, contribuyendo a un mejor entendimiento de los patrones de distribución de pasifloras cultivables y de los potenciales impactos del cambio climático. El marco metodológico propuesto puede proveer importantes herramientas para la gestión y aprovechamiento sostenible de los cultivos de maracuyá, granadilla y cholupa en regiones tropicales para enfrentar los desafíos del cambio climático.

\section{CONTRIBUCIÓN DE LA AUTORÍA}

Primer autor: metodología, investigación, análisis de datos, conceptualización, escritura, borrador original. Segundo autor: análisis de datos, revisión, discusión de resultados y edición. Tercer autor: adquisición de recursos, administrador del proyecto, supervisión, conceptualización, revisión y edición.

\section{AGRADECIMIENTOS}

Los autores agradecen al Ministerio de Ciencia Tecnología e Innovación [Minciencias] y al Fondo Nacional de Financiamiento para la Ciencia, Tecnología y la Innovación "Francisco José de Caldas" por financiar la estancia postdoctoral de A. M. Munar desarrollada en la Corporación Centro de Desarrollo Tecnológico de las Pasifloras de Colombia [CEPASS]. Este estudio se deriva de la investigación postdoctoral: "Potenciales impactos del cambio climático en la dinámica hidrológica y en los patrones de distribución de Passifloras en la cuenca alta del río Magdalena, utilizando modelación hidrológica/modelación de distribución de especies, dados in-situ, 
técnicas de percepción remota y proyecciones de cambio climático AR5 - IPCC" aprobada por Minciencias en la Convocatoria 848/2019.

\section{LITERATURA CITADA}

Araújo, M. H., Silva, I. C., Oliveira, P. F., Barreto, A. R. R., Konno, T. U. P., Esteves, F. A, Barth, T., Aguiar, F., Lopes, N., Dermenjian, R. K., Guimarães, D. O., Leal, I. C. R., Lasunskaia, E. B. \& Muzitano, M. F. (2017). Biological activities and phytochemical profile of Passiflora mucronata from the Brazilian Restinga. Revista Brasileira de Farmacognosia, 27(6), 702-710.https://doi. org/10.1016/j.bjp.2017.07.005

Bartomeus, I., Park, M. G., Gibbs, J., Danforth, B. N., Lakso, A. N. \& Winfree, R. (2013). Biodiversity ensures plant-pollinator phenological synchrony against climate change. Ecology letters, 16(11), 1331-1338. https://doi.org/10.1111/ele.12170

Bejarano, P. A. D. y Ibarra, F. D. M. M. (2018). Impacto de la variabilidad climática y de los sistemas agrarios en el cultivo de granadilla (Passiflora ligularis Juss) de Oxapampa, Pasco, Perú. Biotempo, 15(1), 41-48.

Bezerra, A. D. M., Pacheco, A. J., Bomfim, I. G. A., Smagghe, G. \& Freitas, B. M. (2019). Agricultural area losses and pollinator mismatch due to climate changes endanger passion fruit production in the Neotropics. Agricultural systems, 169, 49-57. https:// doi.org/10.1016/j.agsy.2018.12.002

Burkle, L. A., Marlin, J. C. \& Knight, T. M. (2013). Plant-Pollinator Interactions over 120 years: Loss of species, Co-Occurrence, and Function. Science, 339(6127), 1611-1615. https://doi.org/10.1126/science.1232728

Collevatti, R. G., Nabout, J. C. \& DinizFilho, J. A. F. (2011). Range shift and loss of genetic diversity under climate change in Caryocar brasiliense, a Neotropical tree species. Tree Genetics \& Genomes, 7(6), 1237-1247. https://doi.org/10.1007/ s11295-011-0409-z

Collevatti R. G., Lima-Ribeiro M. S., Terribile L. C., Guedes L. B. S., Rosa F. F. \& Telles M. P. C. (2014). Recovering species demographic history from multi-model inference: the case of a Neotropical savanna tree species. BMC Evolutionary Biology 14(213). https://doi. org/10.1186/s12862-014-0213-0

Collins, W. J., Bellouin, N., DoutriauxBoucher, M., Gedney, N., Halloran, P., Hinton, T., Hughes, J., Jones. C. D., Joshi, M., Liddicoat, S., Martin, G., Connor, F. O., Rae, J., Senior, C., Sitch, C., Toterdell, I., Wilthshire, A \& Woodward, S. (2011). Development and evaluation of an Earth-System modelHadGEM2. Geoscientific Model Development, 4(4), 1051-1075. https://doi.org/10.5194/ gmd-4-1051-2011

Donoghue, M. J. \& Edwards, E. J. (2014). Biome Shifts and Niche Evolution in Plants. Annual Review of Ecology, Evolution, and Systematics, 45, 547572. https://doi.org/10.1146/annurevecolsys-120213-091905

Elias, M. A. S. , Borges, F. J. A., Bergamini, L. L., Franceschinelli, E. V. \& Sujii, E. R. (2017). Climate change threatens pollination services in tomato crops in Brazil. Agriculture, Ecosystems \& Environment, 239, 257-264. https://doi.org/10.1016/j. agee.2017.01.026

Espíndola, A., Pellissier, L., Maiorano, L., Hordijk, W., Guisan, A. \& Alvarez, N. (2012). Predicting present and future intra-specific genetic structure through niche hindcasting across 24 millennia. Ecology Letters, 15(7), 649-657. DOI: https://doi.org/10.1111/ j.1461-0248.2012.01779.x

Farr, T. G., Rosen, P. A., Caro, E., Crippen, R., Duren, R., Hensley, S., Kobrick, M., Paller, M., Rodríguez, E., Roth Ladislav., Seal, D., Shaffer, S., Shimada, J., Umland, J., Werner, M., Oskin, M., Burbank, D. \& Alsdorf, D. (2007). The shuttle radar topography mission. Reviews of geophysics, 45(2). https://doi. org/10.1029/2005RG000183

Fernandes, F. F., Esposito, M. P., Gonçalve, M. R. , Cardoso-Gustavson, P., Furlan, C. M., Hoshika, Y., Carrari, E., Magni, G., Domingos, M. \& Paoletti, E. (2019). The passion fruit liana (Passiflora edulis Sims, Passifloraceae) is tolerant to ozone. Science of The Total Environment, 656, 1091-1101. https://doi. 
org/10.1016/j.scitotenv.2018.11.425

Fick, S. E. \& Hijmans, R. J. (2017). WorldClim 2: new 1-km spatial resolution climate surfaces for global land areas. International journal of climatology, 37(12), 4302-4315. https://doi.org/10.1002/ joc. 5086

Fischer, G., Casierra-Posada, F. y Piedrahíta, W. (2009). Ecofisiología de las especies pasifloráceas cultivadas en Colombia. En D. Miranda, G. Fischer, C. Carranza, S. Magnitskiy, F. Casierra-Posada, W. Piedrahíta, L. E. Flórez (Eds.). Cultivo, poscosecha y comercialización de las pasifloráceas en Colombia: maracuyá, granadilla, gulupa y curuba (pp. 45-67). Sociedad Colombiana de Ciencias Hortícolas.

Fischer, G. \& Miranda, D. (2021). Review on the ecophysiology of important Andean fruits: Passiflora L. Revista Facultad Nacional de Agronomía Medellín, 74(2), 94719481. https://doi.org/10.15446/rfnam. v74n2.91828

Giannini, T. C., Acosta, A. L., Silva, C. I., Alves, P. E., Imperatriz-Fonseca, V. L. \& Saraiva, A. M. (2013). Identifying the areas to preserve passion fruit pollination service in Brazilian Tropical Savannas under climate change. Agriculture, ecosystems \& environment, 171, 39-46. https://doi. org/10.1016/j.agee.2013.03.003

Gobernación del Huila. (2020). Evaluación Agropecuaria del Huila 2019. https://www. huila.gov.co/agricultura-y-mineria/

Intergovernmental Panel on Climate Change. (2013a). Climate Change 2013: the physical basis. https://www.ipcc.ch/site/ assets/uploads/2018/02/WG1AR5_all_final. pdf

Intergovernmental Panel on Climate Change. (2013b). Climate Change 2013: The Physical Science Basis. Cambridge University Press. https://doi.org/10.1017/ CBO9781107415324

Khubaib, N., Asad, S. A., Khalil, T., Baig, A., Atif, S., Umar, M., Kropp, J., Pradhan, P. \& Baig, S. (2021). Predicting areas suitable for wheat and maize cultivation under future climate change scenarios in Pakistan. Climate Research, 83, 15-25. https://doi. org/10.3354/cr01631

Kjøhl, M., Nielsen, A. \& Stenseth, N. C. (2011). Potential effects of climate change on crop pollination. http://www.fao.org/3/ i2242e/i2242e00.pdf

Kogo, B. K., Kumar, L., Koech, R. \& Kariyawasam, C. S. (2019). Modelling Climate Suitability for Rainfed Maize Cultivation in Kenya Using a Maximum Entropy (MaxENT) Approach. Agronomy, 9(11), 727. https:// doi.org/10.3390/agronomy9110727

Kumar, P. (2012). Assessment of impact of climate change on Rhododendrons in Sikkim Himalayas using Maxent modelling: limitations and challenges. Biodiversity and Conservation, 21(5), 1251-1266. https://doi. org/10.1007/s10531-012-0279-1

Läderach, P., Ramirez-Villegas, J., Navarro-Racines, C., Zelaya, C., MartinezValle, A. \& Jarvis, A. (2017). Climate change adaptation of coffee production in space and time. Climatic Change, 141(1), 47-62. https://doi.org/10.1007/s10584-016-1788-9

Loaiza, I. D. y Mesa, N. (2019). Evaluación de déficit hídrico en variantes somaclonales de maracuyá (Passiflora edulis var. Flavicarpa Deneger), usando mediciones morfométricas. Revista de la Asociación Colombiana de Ciencias Biológicas, 1(31), 56-60. https://www.revistaaccb.org/r/index. php/accb/article/view/182/171

Lobell, D.B., Schlenker, W. \& CostaRoberts, J. (2011). Climate trends and global crop production since 1980. Science, 333(6042), 616-620. https://doi. org/10.1126/science.1204531

Ministerio de Agricultura y Desarrollo Rural. (2018). Cadena de pasifloras 2018. https://sioc.minagricultura.gov.co/ Pasifloras/Documentos/2018-09-30\%20 Cifras\%20Sectoriales.pdf

Melgarejo, L. M. Granadilla (Passiflora ligularis Juss): Caracterización ecofisiológica del cultivo. Universidad Nacional de Colombia 
Merow, C., Smith, M. J. \& Silander, J. A. (2013), A practical guide to MaxEnt for modeling species' distributions: what it does, and why inputs and settings matter. Ecography, 36(10), 1058-1069. https://doi. org/10.1111/j.1600-0587.2013.07872.x

Montero, M. U. y Laiton, R. (2016). Zonificación Ambiental para un Sistema Agroforestal en la Producción de maracuyá (Passiflora edulis Sims) y cholupa (Passiflora maliformis L.) en dos zonas del departamento de Huila, Colombia: escala 1:100.000 [Tesis de pregrado, Universidad distrital]. Repositorio institucional. https://repository.udistrital. edu.co/bitstream/handle/11349/3312/ MonteroAcostaManuelUlisesyLaitonHerrera Ricardo2016. pdf?sequence =7\&isAllowed $=y$

Moratelli, R., De Andreazzi, C. S., De Oliveira, J. A. \& Cordeiro, J. L. P. (2011). Current and potential distribution of Myotis simus (Chiroptera, Vespertilionidae). Mammalia, 75(3), 227-234. https://doi. org/10.1515/MAMM.2011.028

Moya, W., Jacome, G. \& Yoo, C. (2017). Past, current, and future trends of red spiny lobster based on PCA with MaxEnt model in Galapagos Islands, Ecuador. Ecology and Evolution, 7(13), 4881-4890. https://doi. org/10.1002/ece3.3054

Nichetoolbox (2016). Welcome to NicheToolBox. http://shiny.conabio.gob. mx:3838/nichetoolb2/

Nix, H. A. (1986). A biogeographic analysis of Australian elapid snakes. En R. Longmore. (Ed.). Atlas of Elapid Snakes of Australia (pp. 4-15). Australian Government Publishing Service.

Ocampo, J., Coppens, G., Restrepo, M., Jarvis, A., Salazar, M. \& Caetano, C. (2007). Diversity of Colombian Passifloraceae: biogeography and an updated list for conservation. Biota Colombiana, 8(1), 1-45. https://www.redalyc.org/articulo. oa?id=49180101

Ocampo, J. (2013). Diversity and Distribution of Passifloraceae in the Department of Huila in Colombia. Acta biológica colombiana, 18(3), 511-516.
Ocampo, J., Urrea, R., Salazar, M., Hernández, J. \& Posada, P. (2013). Avances de investigación en el cultivo de maracuyá (Passiflora edulis $f$. flavicarpa Degener). En C. Carranza y J. Ocampo. (Eds.). Congreso Latinoamericano de Pasifloras (pp. 36-44). Corporación Cepass.

Ocampo, J. A., Rodríguez, A., Puentes, A., Molano, Z. y Parra, M. (2015). El cultivo de la Cholupa (Passiflora maliformis L.): Una alternativa para la fruticultura colombiana. Corporación Centro de Desarrollo Tecnológico de las Pasifloras de Colombia.

Osorio, J. A., Martínez, E. P., Clímaco, J., Aguirre, J. E., Vergara, J. A., Luque, N. Y., Rojas, E. D. y Cruz, G. N. (2020). Caracterización sanitaria de los cultivos de granadilla, gulupa y maracuyá en Colombia, con especial referencia a la secadera causada por Fusarium solani f. sp. passiflorae. Agrosavia. https://doi.org/10.21930/ agrosavia.investigation.7403381

Peterson, A. T., Papes, M. \& Soberón, J. (2008). Rethinking receiver operating characteristic analysis applications in ecological niche modeling. Ecological Modelling, 213(1), 63-72. https://doi. org/10.1016/j.ecolmodel.2007.11.008

Pareek, A., Dhankher, O. P. \& Foyer, C. $\mathrm{H}$. (2020). Mitigating the impact of climate change on plant productivity and ecosystem sustainability. Journal of Experimental Botany, 71(2), 451-456. https://doi.org/10.1093/ jxb/erz518

Pecl, G. T., Araújo, M. B., Bell, J. D., Blanchard, J., Bonebrake, T. C., Chen, I. C., Clark, T. D., Colwell, R. K., Danielsen, F., Evengard, B., Falconi, L., Ferrier, S., Frusher, S., García, R. A., Griffis, R. B., Hobday, A. J., Janion-Scheepers, C., Jarzyna, M. A., Jennings, S ... \& Williams, S. E. (2017). Biodiversity redistribution under climate change: Impacts on ecosystems and human well-being. Science, 355(6332). https://doi. org/10.1126/science.aai9214

Petitpierre, B., McDougall, K., Seipel, T., Broennimann, O., Guisan, A. \& Kueffer, C. (2016). Will climate change increase the risk of plant invasions into mountains? Ecological 
Applications, 26(2), 530-544. https://doi. org/10.1890/14-1871

Phillips, S. J., Anderson, R. P. \& Schapire, R. E. (2006). Maximum entropy modeling of species geographic distributions. Ecological modelling, 190(3-4), 231-259. https://doi. org/10.1016/j.ecolmodel.2005.03.026

Phillips, S. J., Anderson, R. P., Dudík, M., Schapire, R. E. \& Blair, M. E. (2017). Opening the black box: an open-source release of Maxent. Ecography, 40(7), 887-893. https:// doi.org/10.1111/ecog.03049

Polce, C., Garratt, M. P., Termansen, M., Ramirez-Villegas, J., Challinor, A. J., Lappage, M. G., Boatman, N. D., Crowe, A., Endalew, A., Potts, S. G., Somerwill, K. E. \& Biesmeijer, J. C. (2014). Climate-driven spatial mismatches between British orchards and their pollinators: increased risks of pollination deficits. Global change biology, 20(9), 2815-2828. https:// doi.org/10.1111/gcb.12577

Restrepo, J. D. \& Syvitski, J. P. M. (2006). Assessing the effect of natural controls and land use change on sediment yield in a major Andean River: the Magdalena drainage basin, Colombia. Ambio, 35(2), 65-74. https://doi.org/10.1579/00447447(2006)35[65:ATEONC]2.0.CO;2

Remya, K., Ramachandran, A. \& Jayakumar, S. (2015). Predicting the current and future suitable habitat distribution of Myristica dactyloides Gaertn. using MaxEnt model in the Eastern Ghats, India. Ecological engineering, 82, 184-188. https://doi. org/10.1016/j.ecoleng.2015.04.053

Rosenzweig, C., Elliott, J., Deryng, D., Ruane, A. C., Müller, C., Arneth, A., Boote, K. J., Folberth, C., Glotter, M., Khabarov, N., Neumann, K., Piontek, F., Pugh, T. A. M., Schmid, E., Stehfest, E., Yang, H. \& Jones, J. W. (2014). Assessing agricultural risks of climate change in the 21st century in a global gridded crop model intercomparison. Proceedings of the National Academy of Sciences, 111(9), 3268-3273. https://doi. org/10.1073/pnas. 1222463110

Scanes, C. G. (2018). Human activity and habitat loss: destruction, fragmentation, and degradation. En C. G. Scanes y S. R. Toukhsati. (Eds.). Animals and human society (pp. 451-482). Academic Press. https://doi. org/10.1016/B978-0-12-805247-1.00026-5

Scherer, C. C. (2014). Conservação filogenética de nicho climático para espécies do gênero Passiflora L. (Passifloraceae) com ocorrência no Brasil [Tesis de maestria, Universidad Federal de Paraná]. Biblioteca digital: Teses \& Dissertações.

https://acervodigital.ufpr.br/bitstream/ handle/1884/36262/R\%20-\%20D\% 20 -\%20CAROLINA\%20COELHO\%20SCHERER. pdf? sequence $=1$ \&isAllowed $=y$

Settele, J., Bishop, J. \& Potts, S. G. (2016). Climate change impacts on pollination. Nature Plants, 2(16092), 1-3. https://doi. org/10.1038/nplants.2016.92

Sharma, S., Arunachalam, K., Bhavsar, D. \& Kala, R. (2018). Modeling habitat suitability of Perilla frutescens with MaxEnt in Uttarakhand-A conservation approach. Journal of Applied Research on Medicinal and Aromatic Plants, 10, 99-105. https://doi. org/10.1016/j.jarmap.2018.02.003

Shukla, P. K., Baradevanal, G., Rajan, S. \& Fatima, T. (2020). MaxEnt prediction for potential risk of mango wilt caused by Ceratocystis fimbriata Ellis and Halst under different climate change scenarios in India. Journal of Plant Pathology, 102, 765-773. https://doi.org/10.1007/s42161-02000502-9

Stockwell, D. (1999). The GARP modelling system: problems and solutions to automated spatial prediction. International Journal of Geographical Information Science 13(2), 143-158. https://doi. org/10.1080/136588199241391

Su, P., Zhang, A., Wang, R., Wang, J., Gao, Y. \& Liu, F. (2021). Prediction of Future Natural Suitable Areas for Rice under Representative Concentration Pathways (RCPs). Sustainability, 13(3), 1580. https:// doi.org/10.3390/su13031580

Sun, J., Cheng, G. W. \& Li, W. P. (2013). Meta-analysis of relationships between environmental factors and above ground 
biomass in the alpine grassland on the Tibetan Plateau. Biogeosciences, 10, 1707-1715. https://doi.org/10.5194/bg-10-1707-2013

Sutherst, R. W. \& Maywald, G. F. (1985). A computerised system for matching climates in ecology. Agriculture, Ecosystems \& Environment, 13(3-4), 281-299. https://doi. org/10.1016/0167-8809(85)90016-7

Swets, J. A. (1988). Measuring the accuracy of diagnostic systems. Science, 240(4857), 1285-1293. https://doi. org/10.1126/science. 3287615

Thuiller, W., Brotons, L., Araújo, M. B. \& Lavorel, S. (2004). Effects of restricting environmental range of data to project current and future species distributions. Ecography, 27, 165-172. https://doi.org/10.1111/ j.0906-7590.2004.03673.x

United States Agency for International Development. (2014). The US market for passion fruit. http://pdf.usaid.gov/pdf_docs/ PA00KP21.pdf

United States Geological Survey. (2005). Shuttle Radar Topography Mission. https:// www.usgs.gov/centers/eros/science/usgseros-archive-digital-elevation-shuttle-radartopography-mission-srtm

Vanbergen, A. J., \& the Insect Pollinators Initiative. (2013). Threats to an ecosystem service: pressures on pollinators. Frontiers in Ecology and the Environment, 11(5), 251259. https://doi.org/10.1890/120126

Yi, Y.., Cheng, X., Yang, Z. \& Zhang, S. (2016). Maxent modeling for predicting the potential distribution of endangered medicinal plant (H. riparia Lour) in Yunnan, China. Ecological Engineering, 92, 260-269. https:// doi.org/10.1016/j.ecoleng.2016.04.010
Yuan, H., Wei, Y., \& Wang, X. (2015). Maxent modeling for predicting the potential distribution of Sanghuang, an important group of medicinal fungi in China. Fungal Ecology, 17, 140-145. https://doi.org/10.1016/j. funeco.2015.06.001

Wei, B., Wang, R., Hou, K., Wang, X., \& Wu, W. (2018). Predicting the current and future cultivation regions of Carthamus tinctorius $L$. using MaxEnt model under climate change in China. Global Ecology and Conservation, 16, 1-12. https://doi.org/10.1016/j.gecco.2018. e00477

Werneck, F. P., Costa, G. C., Colli, G. R., Prado, D. E., \& Sites J. W. (2011). Revisiting the historical distribution of Seasonally Dry Tropical Forests: new insights based on palaeodistribution modelling and palynological evidence. Global Ecology and Biogeography, 20(2), 272-288. https://doi. org/10.1111/j.1466-8238.2010.00596.x

Wohlmuth, H., Penman, K. G., Pearson, T., \& Lehmann, R. P. (2010). Pharmacognosy and chemotypes of passionflower (Passiflora incarnata L.). Biological and Pharmaceutical bulletin, 33(6), 1015-1018. https://doi. org/10.1248/bpb.33.1015

Woodward, F. I. (1987). Climate \& Plant Distribution. Cambridge University Press.

Zhang, X., Li, G., \& Du, S. (2018). Simulating the potential distribution of Elaeagnus angustifolia L. based on climatic constraints in China. Ecological Engineering, 113, 27-34. https://doi.org/10.1016/j. ecoleng.2018.01.009

Conflicto de intereses

Los autores declaran no tener ningún conflicto de intereses.

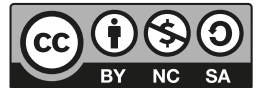

Licencia de Creative Commons

Revista de Investigación Agraria y Ambiental is licensed under a Creative Commons

Reconocimiento - No Comercial - Compartir Igual 4.0 Internacional License. 
